EPiC Series in Computing
Volume 66, 2019, Pages 68-76
Proceedings of 6th International OM-
NeT++ Community Summit 2019

\title{
Everything You Always Wanted to Know About BGPv4 in OMNeT++ But Were Afraid to Ask
}

\author{
Vladimír Veselý ${ }^{1 *}$, Marcel Marek$^{2}$, Kamil Jeřábek ${ }^{1}$, and Adrián Novák ${ }^{3}$ \\ 1 Faculty of Information Technology, Brno University of Technology, Brno, Czech Republic \\ \{veselyv, ijerabek\}@fit.vutbr.cz \\ 2 The Faculty of Mathematics and Natural Sciences, University of Oslo, Oslo, Norway \\ marcelma@ifi.uio.no \\ 3 Faculty of Information Technology, Brno University of Technology, Brno, Czech Republic \\ adriannovak94@gmail.com
}

\begin{abstract}
Border Gateway Protocol is one and only one exterior gateway protocol for routing between autonomous systems, which basically glues the Internet together. This paper outlines BGPv4 theory and its (re)implementations in OMNeT++ discrete event simulator. This effort extends IPv6 capabilities of INET4 framework and improves the accuracy of relevant simulation models.
\end{abstract}

\section{Introduction}

The Internet consists of autonomous systems (AS), where each one represents independent collection of devices and networks under the common administrative domain. AS are uniquely identified by autonomous system number (ASN, which is 16 or 32 bits long identifier [1]). Inside the AS, interior gateway protocols (such as distance-vector or link-state) are guaranteeing level of convergence, which reflects changes fast. Outside the AS, exterior gateway protocols (EGPs) maintains routing for default-free zone (DFZ, i.e., the backbone of the Internet). AS is treated as a single undivided entity from the perspective of global routing. EGPs consider the route as the list of ASN (hence, these protocols are often referred as path-vector routing protocols). EGP or path-vector are remarked as categories but they in fact currently contain only a single representative - Border Gateway Protocol (BGP).

Even though RFC 2460 [2] is more than 20 years old, IPv6 adoption is still taking time to gain significant momentum. It depends on the point of view [3] how to quantify IPv6 penetration ( $8.5 \%$ of web sites, $15.8 \%$ of mail servers, please see [4] for details), but it is safe to say that $\operatorname{IPv} 6$ availability in 2019 is probably lower than authors (and other proponents) of IPv6 would hope for. However, IPv6 is still the one and only widely accepted option that is going to substitute $\mathrm{IPv} 4$ and its addressing limitations. Hence, various service providers (including ISPs, content cachers, service deliverers and many others) are working on necessary

${ }^{*}$ Corresponding author OMNeT Summit 2019 (EPiC Series in Computing, vol. 66), pp. 68-76 
changes in order to prepare their infrastructure and applications for IPv6. A significant part of this effort is invested into the routing of IPv6 traffic, which would be impossible without appropriate routing protocols. Many protocols support IPv6 address family - OSPFv3, EIGRP, IS-IS, Babel, RIPng, M-BGP. However, only some of them - namely OSPF and BGP - receive recognition as a standard tools of LAN/WAN design [5].

General idea driving the development of simulation software is to create a tool which: a) imitates a system behavior under various conditions; and b) subsequently generates accurate and reliable results. We believe that OMNeT++ is such a tool and its INET framework is great for simulations of computer networks. Primary motivation of this paper is to enhance IPv6 support within INET4 framework. We planned to achieve this goal by improving functionality of widely used routing protocol BGP. This effort is a part of long-term running project (called ANSA) at Brno University of Technology, which focuses on implementation of various computer networking protocols for $\mathrm{OMNeT}++$.

The paper has the following structure. Section 2 explains basic theory behind BGP protocols. Section 3 describes the current state in the frame of INET4 and outlines design and implementation notes of the source contribution. Section 4 demonstrates correct behavior of simulation models on scenarios also compared with referential Cisco implementation of BGP. Section 5 summarizes achievements of this paper together with a brief overview of future plans.

\section{Principles}

This section briefly overviews existing BGP protocol and its operational principles.

BGP is a path-vector routing protocol, which distributes classless routing information (mostly) between AS. BGP is useful for dual-homed or (dual-)multi-homed AS, where routing information needs to be propagated to the DFZ. BGP offers summarization and authentication (special TCP option with MD5-HMAC).

BGP peers or neighbors are routers running instance of BGP and exchanging routes with each other. BGP peering comes with two modes: a) external BGP (eBGP), where routers are in different autonomous systems; and b) internal BGP (iBGP), where both routers are in the same AS. In the case of eBGP, peers are usually adjacent (one hop away) and exchange all routing information. However, iBGP peers exchange only directly connected networks (i.e., prefixes originating in the same AS). This restriction works as built-in protection against routing loops for iBGP that also yields full-mesh connectivity between iBGP peers.

BGP transfers messages reliably with the help of TCP as the transport protocol. Hence, BGP was assigned with well-known port 179 as the application over TCP. Despite the existence of multiple versions of BGP, only the newest one BGPv4 is being used (see RFC 4271 [6]).

However, numerous RFCs are extending protocol capabilities (see RFC 4760 [7]) of this version, and they offer MPLS, VPN, multi-address family, or multicast support. The BGP models network as a graph, where vertices represent autonomous systems and edges represent connections between them. These vertices include a list of destination networks contained within AS. Series of edges then represent suitable paths to reach destinations, where paths include various attributes such as next-hop, AS-path (a list of autonomous system numbers), local preference (announced exit point from AS), multi-exit discriminator (preferred entry point to AS) and others. By (re)configuring these attributes, BGP can enforce inbound and outbound routing policies that affect how traffic is routed to/from/via the AS.

BGP protocol uses the following message types:

- Open as the initial message, which is sent after TCP connection is established between 
peers;

- KeepAlive that is periodically sent as a heartbeat, which signals that peer is working correctly. KeepAlive is sent every $60 \mathrm{~s}$ by default and neighbor is willing to wait generally three times more (so-called HoldDown timer, which is $180 \mathrm{~s}$ by default) before declaring the router down;

- Update message carries routing information for a single path (unique route across AS), which includes attributes (mentioned above), the list of reachable destination networks (using this path) and optionally the list of withdrawn destinations;

- Notification is generated whenever some problem with BGP occurs and terminates BGP peering. Notification contains error code and more verbose error description.

After the initial synchronization, BGP peers send only incremental updates (i.e., new or withdrawn routes) because this approach is more scalable than a periodic exchange of the whole routing tables (which in the case of DFZ router with many peers may be GBs of routing data).

\section{Implementation}

This section discusses current development state of INET framework pursuant to abovementioned routing protocols. Moreover, the section briefly covers the design of enhanced simulation model for BGPv4.

\subsection{INET}

Open-source framework INET helps academia and industry to simulate computer networks. INET model library spans through the whole network stack and includes models for Ethernet, WiFi, IPv4/IPv6, TCP/UDP/SCTP and plethora of routing and application protocols. ANSAINET is a fork of INET that further extends capabilities and available models of the original framework. ANSAINET feature-set focuses on the simulation of wired enterprise networks. We have decided to revisit the source base of INET, namely INET4 and more specifically, inet-4.0.0-576b2dc.

INET partially implements BGPv4 since 2010 (code by Helene Lageber). We identified the following list of problems in above-mentioned version of INET4:

P1) Peer is unable to advertise more than one network prefix to its neighbor. Update message contains only one network prefix, and a neighboring peer is unable to process more prefixes. Hence, it is currently impossible to announce more network prefixes between peers.

P2) The current implementation does not support IPv6 prefixes, only IPv4.

P3) AS Path appending adds ASN even in cases of iBGP, which should not happen. Moreover, current iBGP peers are willing to exchange all routing information (both learned and directly connected prefixes), which violates an important operational condition of BGP and leads to routing instabilities in certain topologies.

P4) If a router has active eBGP peering, then it is impossible to form any iBGP with other potential routers.

P5) ConnectRetry timer is not being reset correctly. When this timer expires, BGP routers lose TCP connection and stop being peers.

P6) Implementation (namely the state of BGP peering) is unable to react on link flapping. It is neither able to tear down the neighborship, nor to re-establish it. 
P7) Notification message is not implemented at all, and there are inconsistencies in the finite state machine of BGP operation.

The main goal of our contribution is to support IPv6 in BGPv4 implementation in OM$\mathrm{NeT}++$. Nevertheless, we have also decided to amend all issues and problems discovered during the assessment of source code base.

\subsection{Design}

BGPv4 is designed as a simple module named BGP and it is available in the following folder src/inet/routing/bgpv4.

We extended appropriate message definition files with new headers and fields - namely multiprotocol capability indicators, network (un)reachability and address-family specifiers, in order to address P1, P2 and P7. Moreover, we have added appropriate version of all BGP messages for IPv6 address-family (if IPv6 is used as a carrier protocol for peering).

BGP tracks the status of TCP connection and neighborship with every peer in BGPSession. We extended this abstract data structure with information whether the neighborship leverages IPv4 or IPv6 (resolving problem P2) and which type of peering is employed (resolving P4).

We have also implemented all missing parts of the finite state machine in BgpFsm as well as event listeners in order to deal with P2, P3, P5 and P6. Our contribution covers all standard BGP states and appropriate transitions between them:

- Idle - Beginning state of any neigborship and a fallback state for BGP related errors.

- Connect - Router creates TCP connection with its peer, or more accurately, the peer with higher IP address initiates TCP connection while the other peer listens for it.

- Active - If TCP connection can not be established, the router resets ConnectRetry timer during this state and repeats connection attempt. This state is used as a fallback for TCP related errors.

- OpenSent - TCP connection was successfully established (i.e., communicating peers conducted 3-way handshake). The Open message is sent to validate the basic settings of BGP peering. Following properties must match between potential peers: BGP version, unique router identifier, autonomous system numbers, peering IP addresses, optionally authentication or time-to-live value.

- OpenConfirm - The router received Open also from a neighbor and now it waits for first KeepAlive, which states that basic settings is fine. To avoid endless waiting for response, HoldDown timer is started. If KeepAlive is received, then the peer is ready to exchange network prefixes.

- Established - Peers exchange routing information using Updates. HoldDown is reset with every KeepAlive or Update to monitor peer's heartbeat.

Detail description of the implemented finite state machine is beyond the scope of this paper, but illustration with the most important parts is available on the results reproduction webpage accompanying this contribution [8].

We have also created a new XML-based configuration file for BGP module that bootstraps all settings upon initialization of simulation scenario. A new parser was introduced to support the updated XML structure which can specify - interface addressing setup, BGP address-family configuration, neighbor specification, and advertised prefixes. 


\section{Testing}

In this section, we focus on testing of our code extension of BGPv4 in INET framework. The extension covers missing functionality as described above (most notably IPv6 support, more prefixes in Updates, reaction to the failure/recovery). The chosen testing scenario primarily demonstrates and tests multi address-family routing functionality that covers both IPv6 and $\mathrm{IPv} 4$ routing. The behavior of the new simulation model is compared with the referential Cisco implementation (IOS version 15.4), which is compliant with the standard and outlined in [9].

\subsection{Topology}

The testing topology depicted in Figure 1 contains four routers R1-R4. Each router has configured loopback interface Loopback0 with specific IPv4 and IPv6 addresses that can be seen in the Figure 1 above each router. The router R1 is part of AS 100. The routers R2 and R3 are part of AS 200, and internal neighborship is established between them. We use static routing inside AS 200 to simplify the scenario. The router R4 is part of AS 300. External neighborship is established between routers R1-R2 and R3-R4. All routers negotiate their Loopback0 IPv4 and IPv6 addresses.

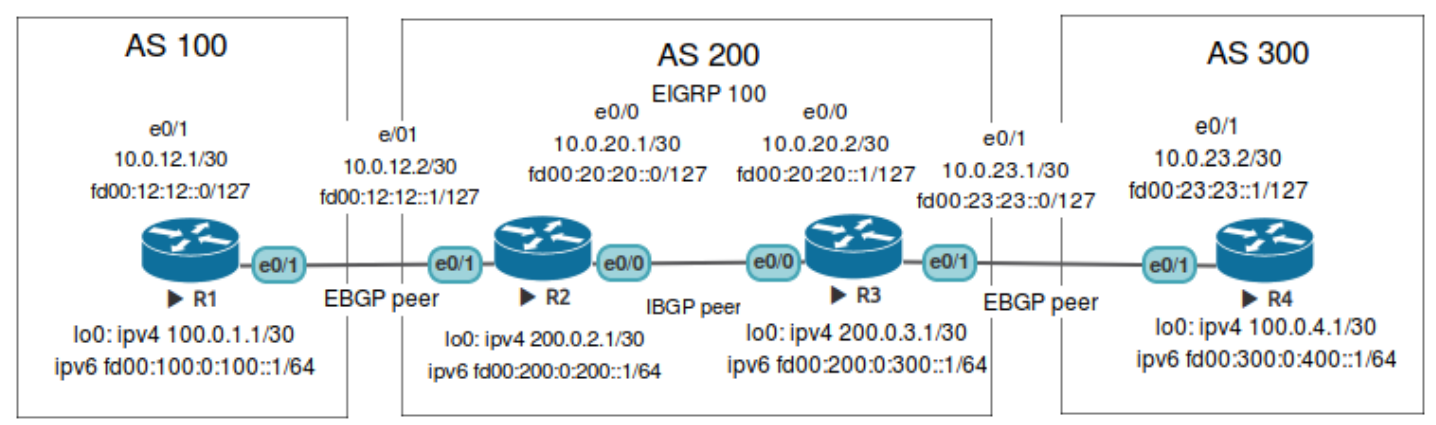

Figure 1: BGP testing topology.

The example can be found in the directory examples/bgpv4/BgpEx1Loopback and it contains three simulation configurations, where for the cause of this paper is relevant the one called LinkDownAndUp.

\subsection{Connection Establishment}

In this part of the scenario, we cover neighborship establishment from the perspective of router R1 communicating with router R2. It is the first connection made in the scenario/experiment. The messages produced during the connection establishment phase are depicted in Table 1 . The table shows comparison of message exchange in simulation and in referential network. The table shows network simulation message exchange in comparison to real network message exchange. The order of messages in the referential implemenetation differs because TCP connections for IPv4 and IPv6 are initiated in parallel.

The establishment phase in the simulation environment goes as follows:

\#1A) At first, router R1 creates a TCP connection for IPv4 to router R2. Router R1 sends Open message with initial BGP information. Router R2 sends a positive response to Open. The KeepAlive message exchange follows. 
\#2A) Router R1 then sends Update message with known routes (in this case only the route to the network of the Loopback0 is negotiated). In response, the router R2 sends Update with its known routes.

\#3A) Router R1 creates another TCP connection for IPv6 to router R2. The BGP message exchange for IPv6 is similar to the $\mathrm{IPv} 4$ one.

\#4A) Router R1 receives only one Update for each TCP connection with information regarding the rest of the network, because the rest of the routers exchange BGP information later then R1 and R2.

More KeepAlive messages follow after but they are not included in Table 1. Timestamps included in this and subsequent tables represent real timestamps as recorded by the simulation log or PCAP file. Therefore, you can observe slight discrepancies because it is hard to align events of the simulated and real control plane. If you focus on message order, then you check the same transition through BGP finite state machine, which ends with the same content of routing tables. Message order may be slightly different because of packet pacing and control plane delay processing in case of the referential implementation.

\begin{tabular}{|c|c|l|c|c|l|}
\hline \multicolumn{3}{|c|}{ Simulation } & \multicolumn{3}{c|}{ Referential implementation } \\
\hline Phase & Time & Message & Phase & Time & Message \\
\hline \#1A & 17.000 & $\rightarrow$ Open & \#1A & 54.621 & $\rightarrow$ Open \\
\#1A & 17.000 & $\leftarrow$ Open & \#3A & 54.622 & $\rightarrow$ Open6 \\
\#1A & 17.000 & $\rightarrow$ KeepAlive & \#1A & 54.623 & $\leftarrow$ Open \\
\#1A & 17.000 & $\leftarrow$ KeepAlive & \#1A & 54.623 & $\rightarrow$ KeepAlive \\
\#2A & 17.000 & $\rightarrow$ Update & \#1A & 54.623 & $\leftarrow$ KeepAlive \\
\#2A & 17.000 & $\leftarrow$ Update & \#3A & 54.626 & $\leftarrow$ Open6 \\
\#3A & 17.500 & $\rightarrow$ Open6 & \#3A & 54.626 & $\leftarrow$ KeepAlive6 \\
\#3A & 17.500 & $\leftarrow$ Open6 & \#2A & 54.629 & $\rightarrow$ Update \\
\#3A & 17.500 & $\rightarrow$ KeepAlive6 & \#3A & 54.630 & $\rightarrow$ KeepAlive6 \\
\#3A & 17.500 & $\leftarrow$ KeepAlive6 & \#2A & 54.630 & $\leftarrow$ Update \\
\#3A & 17.500 & $\rightarrow$ Update6 & \#3A & 54.635 & $\rightarrow$ Update6 \\
\#3A & 17.500 & $\leftarrow$ Update6 & \#3A & 54.635 & $\leftarrow$ Update6 \\
\#4A & 21.000 & $\leftarrow$ Update & & & \\
\#4A & 26.000 & $\leftarrow$ Update6 & & & \\
\hline
\end{tabular}

Table 1: BGP connection establishment messages captured from router R1.

\subsection{Connection Failure}

In this section, we demonstrate the behaviour after a link failure. The link interconnecting router R1 and R2 is removed. Table 2 provides message exchange from the perspective of router R2.

\#1B) KeepAlives from both R1 and R2 do not reach each other. HoldDown timer is set to $180 \mathrm{~s}$ (which is recommended value). KeepAlive messages normally reset the timer. When the timer expires, the router R2 removes peer routes from its routing table. The router R2 immediately generates Update messages and sends them to its peer router R3.

\#2B) The router R3 receives Update for $\mathrm{IPv} 4$, removes the route from its routing table and sends Update to router R4. Similar process repeats also for IPv6.

In the simulation, the last KeepAlive message from R1 to R2 gets through at $t=17.0 \mathrm{~s}$. 
HoldDown timer expired three minutes (i.e., $180 \mathrm{~s}$ ) since then at $t=197.0 \mathrm{~s}$. R2 sends Update to $\mathrm{R} 3$ for $\mathrm{IPv}_{\mathrm{v}}$ and $0.5 \mathrm{~s}$ later Update6 for IPv6. In the referential implementation, the link shuts down at around $t=35 \mathrm{~s}$ relative to the packet capture start. The time gap between Update and Update6 message is that the experiment was running for some time and the timers diverged.

The reason why there is a gap of $50 \mathrm{~s}$ (which is roughly equal to the value of KeepAlive timer) between the two Update is because $\mathrm{IPv}_{\mathrm{v}}$ KeepAlive was received just before the link was shut down. Therefore, the Update6 was sent sooner at $t=165 \mathrm{~s}$ and Update at $t=214 \mathrm{~s}$.

\begin{tabular}{|c|c|c|c|c|c|}
\hline \multicolumn{3}{|c|}{ Simulation } & \multicolumn{3}{c|}{ Referential implementation } \\
\hline Phase & Time & Message & Phase & Time & Message \\
\hline$\# 1 \mathrm{~B}$ & 197.000 & $\rightarrow$ Update & $\# 1 \mathrm{~B}$ & 165.287 & $\rightarrow$ Update6 \\
$\# 1 \mathrm{~B}$ & 197.500 & $\rightarrow$ Update6 & $\# 1 \mathrm{~B}$ & 214.641 & $\rightarrow$ Update \\
\hline
\end{tabular}

Table 2: BGP connection failure messages captured from router R2.

\subsection{Connection Recovery}

In this section, we demonstrate the behaviour after a link becomes available again. The link between router R1 and router R2 is recovered. The router R1 and R2 are in the Idle state. The neighborship establishment between those routers proceeds as described in section 4.2. Table 3 depicts the message exchange both for simulation and referential implementation.

\#1C) The router can send all prefixes in the first Update message. Then only KeepAlive messages are exchanged.

The difference between message exchange described in section 4.2 and here occurs because there is only one Update needed from the router R2. Router R2 has already all the information about the topology.

\begin{tabular}{|c|c|c|c|c|c|}
\hline \multicolumn{3}{|c|}{ Simulation } & \multicolumn{3}{|c|}{ Referential implementation } \\
\hline Phase & Time & Message & Phase & Time & Message \\
\hline$\# 1 \mathrm{~A}$ & 217.000 & $\rightarrow$ Open & $\# 1 \mathrm{~A}$ & 118.576 & $\leftarrow$ Open \\
\hline$\# 1 \mathrm{~A}$ & 217.000 & $\leftarrow$ Open & \#3A & 118.577 & $\leftarrow$ Open 6 \\
\hline \#1A & 217.000 & $\rightarrow$ KeepAlive & $\# 1 \mathrm{~A}$ & 118.578 & $\rightarrow$ Open \\
\hline \#1A & 217.000 & $\leftarrow$ KeepAlive & $\# 1 \mathrm{~A}$ & 118.579 & $\leftarrow$ KeepAlive \\
\hline \#1C & 217.000 & $\rightarrow$ Update & \#1A & 118.579 & $\rightarrow$ KeepAlive \\
\hline \#1C & 217.000 & $\leftarrow$ Update & \#3A & 118.579 & $\rightarrow$ Open 6 \\
\hline \#3A & 222.500 & $\rightarrow$ Open6 & \#3A & 118.581 & $\rightarrow$ KeepAlive 6 \\
\hline \#3A & 222.500 & $\leftarrow$ Open 6 & \#3A & 118.584 & $\leftarrow$ KeepAlive 6 \\
\hline \#3A & 222.500 & $\rightarrow$ KeepAlive & $\# 1 \mathrm{C}$ & 118.585 & $\rightarrow$ Update \\
\hline \#3A & 222.500 & $\leftarrow$ KeepAlive 6 & $\# 1 \mathrm{C}$ & 118.585 & $\leftarrow$ Update \\
\hline$\# 1 \mathrm{C}$ & 222.500 & $\rightarrow$ Update 6 & $\# 1 \mathrm{C}$ & 118.591 & $\rightarrow$ Update 6 \\
\hline$\# 1 \mathrm{C}$ & 222.500 & $\leftarrow$ Update 6 & $\# 1 \mathrm{C}$ & 118.591 & $\leftarrow$ Update 6 \\
\hline
\end{tabular}

Table 3: BGP connection recovery messages captured from router $\mathrm{R} 1$. 


\section{Conclusion}

In this paper, we thoroughly described widely deployed protocol for intra (and in some cases also inter) autonomous system routing - BGPv4. We had revisited and significantly improved simulation models of this protocol within INET framework. We created new test scenarios checking both old and new functionalities. We have validated the functionality of our simulation model against referential implementation. Compared message orders yield: a) same outcomes in routing table content; and b) same transitions through BGP finite state machines.

It is important to support IPv6 by providing implementation in computer network simulators despite scarce deployment of this network protocol in production. We believe that our contribution enhances capabilities of (ANSA)INET framework. Moreover, it makes BGPv4 simulations more accurate and thus more reliable in cases when $\mathrm{OMNeT}++$ is being used for network design validations.

More information about the ANSAINET project is available on the homepage [10]. All source codes (BGPv4 simulation models) can be downloaded from the GitHub repository [11]. Moreover, these contributions are planned for direct migration into official INET framework source base.

This work was supported by the Brno University of Technology organization and by the research grant FIT-S-17-3964 and as part of the OCARINA research project [12], which develops a combined congestion control and routing solution for the Recursive InterNetworking Architecture.

\section{References}

[1] John A. Hawkinson and Tony J. Bates. Guidelines for creation, selection, and registration of an Autonomous System (AS). RFC 1930, March 1996.

[2] Bob Hinden and Steve E. Deering. Internet Protocol, Version 6 (IPv6) Specification. RFC 2460, December 1998.

[3] Matěj Grégr, Tomáš Podermański, and Miroslav Švéda. Measuring quality and penetration of ipv6 services. ICNS'14, pages 96-101, 2014.

[4] Matěj Grégr and Tomáš Podermański. Live statistics - 6lab.cz. http://6lab.cz/ live-statistics, accessed: July 2019.

[5] David Bauer, Murat Yuksel, Christopher Carothers, and Shivkumar Kalyanaraman. A case study in understanding ospf and bgp interactions using efficient experiment design. In 20th Workshop on Principles of Advanced and Distributed Simulation (PADS'06), pages 158-165. IEEE, 2006.

[6] Yakov Rekhter, Susan Hares, and Tony Li. A Border Gateway Protocol 4 (BGP-4). RFC 4271, January 2006.

[7] Tony J. Bates, Ravi Chandra, Yakov Rekhter, and Dave Katz. Multiprotocol Extensions for BGP-4. RFC 4760, January 2007.

[8] Brno University of Technology. OMNeT++ Summit 2019 results reproduction. https://github. com/ANSA/results-reproduction/wiki/OMNeTpp-Summit-2019, accessed: July 2019.

[9] Vinit Jain and Bradley Edgeworth. Troubleshooting BGP: A Practical Guide to Understanding and Troubleshooting BGP. Cisco Press, 2016.

[10] Brno University of Technology. ANSA project. http://ansa.omnetpp.org, accessed: July 2019.

[11] Brno University of Technology. ANSAINET repository. https://github.com/ANSA/inet, accessed: July 2019. 
Everything You Always Wanted to Know About BGPv4 in OMNeT++

Veselý, Marek, Jeřábek and Novák

[12] University of Oslo. OCARINA: Optimizations to compel adoption of rina - department of informatics. https://www.mn.uio.no/ifi/english/research/projects/ocarina, accessed: July 2019 . 